\title{
Nonlinear relaxation and selective polychromatic lasing of confined polaritons
}

\author{
G. Grosso, ${ }^{1}$ S. Trebaol, ${ }^{1}$ M. Wouters, ${ }^{2}$ F. Morier-Genoud, ${ }^{1}$ M. T. Portella-Oberli, ${ }^{1}$ and B. Deveaud ${ }^{1}$ \\ ${ }^{1}$ ICMP, Ecole Polytechnique Fédérale de Lausanne (EPFL), 1015 Lausanne, Switzerland \\ ${ }^{2}$ TQC, University of Antwerp, Universiteislpein 1, 2610 Antwerpen, Belgium
}

(Received 8 May 2014; published 15 July 2014)

\begin{abstract}
Polaritons have often been proposed as promising candidates for the realization of all-optical devices due to the easy manipulation and control of their density and spin. In this paper we present a relaxation mechanism for confined polaritons and its application in a device based on polariton lasing in which the incoming monochromatic beam can be channeled into several polariton lasers at different wavelengths. Polaritons are injected in a $3 \mu \mathrm{m}$ diameter trap by an incoming beam slightly detuned with respect to a confined state, and the resulting optical bistability is then imprinted on the lower confined states through an efficient relaxation mechanism which combines phonon interaction and bosonic stimulation. Above threshold, all the confined states behave like lasers and the onset of coherence is demonstrated by spectral narrowing. Moreover, the initial polarization of the laser is conserved during the relaxation process allowing for spin logic operations. In this paper we demonstrate that, due to the nonlinear behavior, it is possible to switch ON and OFF the lasing from the confined states of the trap by tailoring the input beam. All the experimental findings are validated and very well reproduced in the framework of the generalized Gross-Pitaevskii equation.
\end{abstract}

DOI: 10.1103/PhysRevB.90.045307

PACS number(s): 71.36.+c, 78.67.-n, 71.35.Lk, 42.65.Pc

\section{INTRODUCTION}

In the recent past, several approaches to optical switches have been proposed in the field of excitons in semiconductors. Excitons are electron-hole pairs with the key advantage that they easily couple with light. This peculiar feature brings them to the front line for the realization of all-optical and optoelectronic devices [1,2]. Other appealing features arise when excitons are strongly coupled with light in semiconductor microcavities. The resulting particles are known as exciton polaritons. Polaritons have a very light mass, due to the photonic content, and interact among themselves due to their excitonic part giving rise to spectacular nonlinear effects such as solitons [3] and optical bistability [4,5]. On top of that, the polariton density and spin can be easily manipulated by optical means. The combination of all those unique properties favored the realization of a large variety of devices such as transistors [6-8], ultrafast memories [9], spin switches [10], and led to the observation of Bose-Einstein condensation [11] and polariton lasing [12,13] up to room temperature [14]. The accumulation of particles at the lower energy state, necessary to reach the phase transition, has been achieved so far by means of either nonresonant excitation or parametric scattering amplification. Employing these techniques, the resulting coherent state is governed by the intrinsic nature of the system and the control on the final coherent emissions is still an open challenge. In this paper we report on an efficient relaxation mechanism for polaritons and its application in an innovative device allowing for all-optical logic operations. We take advantage of the one-to-one connection between intensity (polarization) of the incident beam with the density (spin) of the trapped polariton gas to drive multivalued output states in terms of wavelength and polarization. Polariton lasing is demonstrated by linewidth narrowing of the polariton states [15]. The experimental findings are validated in the framework of the generalized Gross-Pitaevskii equation. The paper is organized as follows. In Sec. II the sample and the experimental procedure are described. The physical principles that govern the relaxation mechanism of confined polaritons in the linear regimes are detailed in Sec. III. The polariton behavior in the nonlinear regime is discussed in Sec. IV. In Secs. V and VI we describe respectively the polariton polarization and the working principle of the device based on the properties discussed before. A general conclusion is given in Sec. VII.

\section{EXPERIMENTAL DETAILS}

The device is engineered in a GaAs $\lambda$-microcavity with a single $8 \mathrm{~nm}$ InGaAs quantum well giving a Rabi splitting of $3.5 \mathrm{meV}$. Polariton traps, called mesas, are obtained by etching the cavity before growing the top mirror, constituted of a distributed Bragg reflector. The reshaping of $\Delta \lambda$ of the photon wavelength [see Fig. 1(a)] results in a confining potential of $9 \mathrm{meV}$ with respect to the 2D cavity giving rise to discretized energy states [16]. Figure 1(b) shows the calculated photonic modes inside a $3 \mu \mathrm{m}$ quasicircular mesa [17]. Five confined states (CSO-4) appear inside the trap (blue lines) at different energies and with different momentum distribution. At energies greater than the confinement depth, the photonic dispersion shows the typical parabolic shape of planar microcavities. The detuning $\left(\delta_{C S, j}=E_{C S, j}-E_{X}\right)$ of the confined photon states inside the mesa with respect to the exciton is $\delta_{C S 3}=+2 \mathrm{meV}, \delta_{C S 1}=-1.7 \mathrm{meV}$, and $\delta_{G S}=-3.7 \mathrm{meV}$, being $E_{X}=1484.5 \mathrm{meV}$. The sample is held in a cold-finger cryostat at liquid helium temperature. Figure 1(c) shows the experimental polariton dispersion of the system in the linear regime. The slightly elliptical shape of the mesa breaks the rotational symmetry of the system and lifts the degeneracy of the confined states $C S 1$ and $C S 2$ creating two new eigenvalues for each of them [18]. It is worth noting that all the photonic modes of the mesa are coupled with the exciton at different detuning. Therefore the resulting confined polariton modes have different lifetimes due to the diverse photonic and excitonic contents. In Fig. 1(c) we have assigned the name $S j$ to some states that will play a key role in the following text. In particular, $S 0$ corresponds to the polariton 

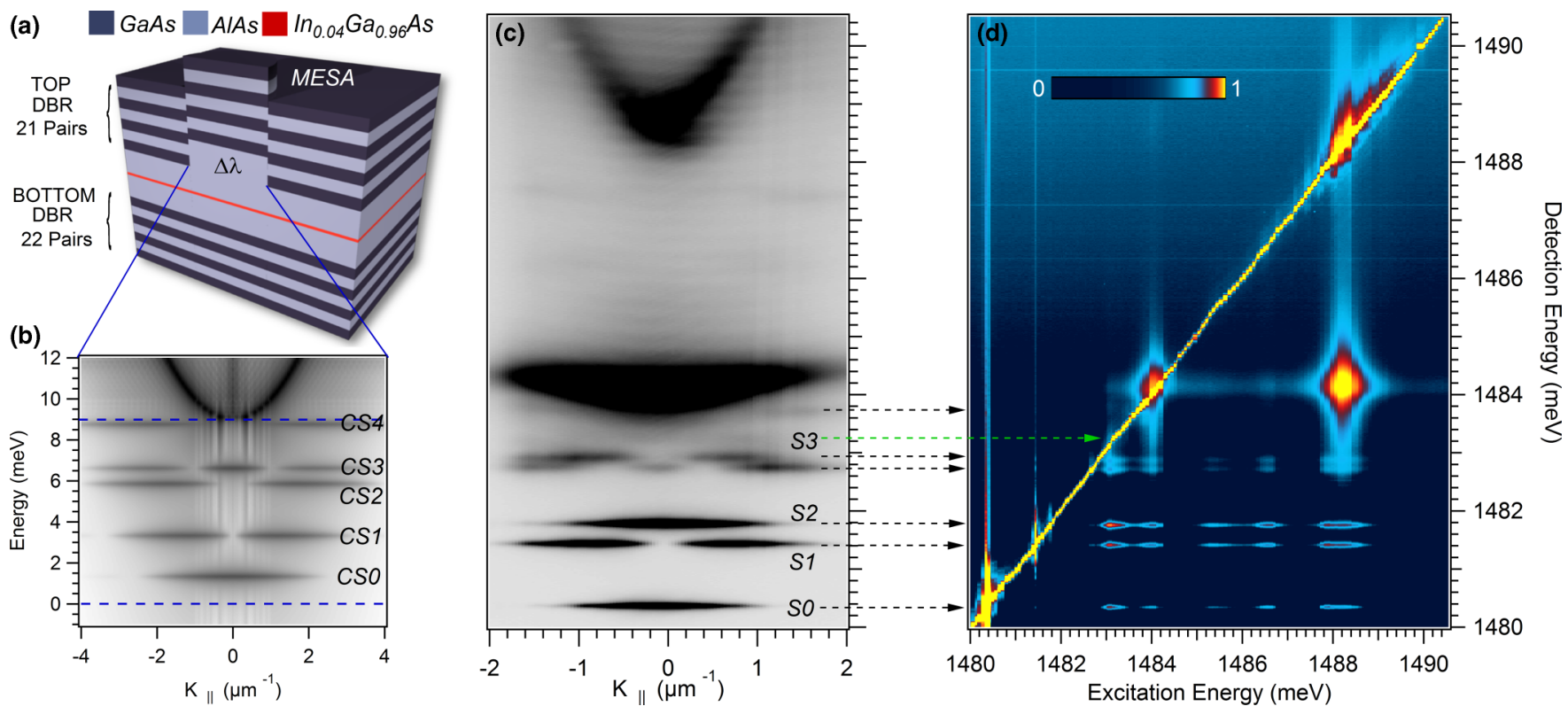

FIG. 1. (Color online) (a) Detailed structure of the semiconductor microcavity and the engineered photonic potential (mesa). (b) Numerical simulation of the confined photonic modes (CSO-CS4) inside the mesa. The blue lines are a guide for the eyes representing the energy of the confining potential. (c) Experimental dispersion for nonresonant excitation. The dispersion shows the upper and lower polariton branches at strong positive detuning $\left(\delta_{2 D}=4 \mathrm{meV}\right)$ and the lower confined states of a $3 \mu \mathrm{m}$ diameter mesa. The states considered in the working principle of the device are labeled as $S_{j}$ with $j=0,1,2,3$. (d) Polariton emission (right axis) as a function of the excitation energy (bottom axis) for $P=100 \mu \mathrm{W}$.

state resulting from the coupling with the exciton of the photonic mode $C S 0 . S 1$ and $S 2$ are the polariton eigenstates related to $C S 1$ and split because of the elliptic shape of the mesa. $S 3$ results from $C S 3$. The two states resulting from $C S 2$ and the one resulting from $C S 4$ are not explicitly mentioned in the figure because they are not relevant for the discussion. The exciton content is found to be $87 \%, 28 \%$, and $14 \%$ for $S 3$, $S 2-S 1$, and $S 0$, respectively. This results in a lifetime for $S 3$ which is an order of magnitude higher than $S 0$.

The mesa is excited at $k_{\|}=0$ with a frequency stabilized continuous-wave Ti:Sapph laser with a Gaussian profile of FWHM $\sim 10 \mu \mathrm{m}$, and it is prepared with circular polarization by using a quarter-wave plate. In the detection side the recorded polarization can be selected with an extinction ratio of the analyzer system of $99.6 \%$. The emission is collected by means of a 0.5 NA microscope objective in transmission configuration. The polariton emission is then spectrally resolved and finally recorded with a high-dynamical-range CCD camera. For the photoluminescence excitation (PLE) measurements, discussed in the next section, the excitation energy is varied with steps of $25 \mu \mathrm{eV}$. In the excitation side the power is tuned by means of motorized stages.

\section{POLARITON RELAXATION IN THE LINEAR REGIME}

The relaxation mechanism inside the mesa is studied using photoluminescence excitation in the linear regime $(P=100 \mu \mathrm{W})$. Figure 1(d) shows the PL spectra (right axis) as a function of the excitation energy (bottom axis) for circular polarization of the excitation. The sharp oblique line represents the laser which is tuned over a range of $\sim 10 \mathrm{meV}$, going from the lower branch of the confined states to the 2D upper polaritons. The PL emission due to resonant excitation is stronger for the lower confined states (S0-2) and almost no emission is observed for $S 3$ as expected from its high excitonic content. This latter feature governs also the relaxation mechanism. When exciting $S 1$ and $S 2$ no relaxation toward $S 0$ occurs. On the contrary, a strong increase appears when exciting $S 3$. When the laser is resonant with this state, the PL emission is observable from $S 0, S 1$, and $S 2$. This behavior is related to the different polariton lifetime of the confined states due to the different excitonic contents. Despite the large energy separation between the polariton states, the phonon assisted relaxation can occur due to the relatively long lifetime of $S 3$ and to the enhanced overlap between the phonon and the confined polariton wave function [19]. It is possible to estimate the polariton distribution along the confined states from the intensity emission of the mesa extracted from the PLE map in the case of resonant excitation of the $S 3$ state. The higher emission of the states $S 2$ and $S 1$ with respect to $S 0$ confirms that the relaxation is mainly assisted by phonon scattering, which is more efficient for a small energy difference between the initial and the final state.

The same PLE experiment has been performed for different mesas at different detunings in order to exclude other possible causes for the efficient relaxation observed from the states with high excitonic content. At more negative detuning the exciton content of $S 3$ decreases and also its relaxation efficiency. In those cases the relaxation from $S 4$ becomes more efficient than $S 3$ despite the unfavorable momentum distribution. At more positive detuning, the higher confined states of the lower branch overlap to the exciton mode and thus to the $2 \mathrm{D}$ polaritons. Therefore states as $S 4$ and $S 3$ cannot be 
distinguished anymore. Anyway, $S 2$ and $S 1$ get more excitonic and some relaxation can be observed towards $S 0$.

\section{NONLINEAR REGIME}

The main idea of this paper is to exploit the efficient relaxation from $S 3$ and imprint its strong nonlinear behavior onto the lower states. Polaritons are selectively injected in the center of the mesa with a momentum $k_{\|}=0$. Therefore the relaxation is predominant toward confined states with a wave function peaked around $k_{\|}=0$. Experiments in micropillars have shown that relaxation prefers states with an antinode of the wave function which coincides with the excitation profile [15]. In order to create a bistable behavior with a nonlinear optical jump, the pump laser is slightly detuned above the state $S 3(\delta=0.3 \mathrm{meV})$. Indeed, $S 3$ displays a nonlinear optical bistability due to polariton-polariton interactions $[4,5,20]$.

We use a quasinonresonant excitation and we focus on the behavior of the polariton population in the lower confined states. An energy resolved intensity map of the mesa emission as a function of the pump power is plotted in Fig. 2(a) in saturated color scale. The laser beam is prepared in circular polarization $\left(\sigma^{+}\right)$and the emission is recorded for cocircular polarization. The normalized intensity for the polariton states is then extracted and displayed in Fig. 2(b). At low power, the intensity of $S 3$ follows the expected linear behavior. The increasing density leads to a blueshift of the polariton
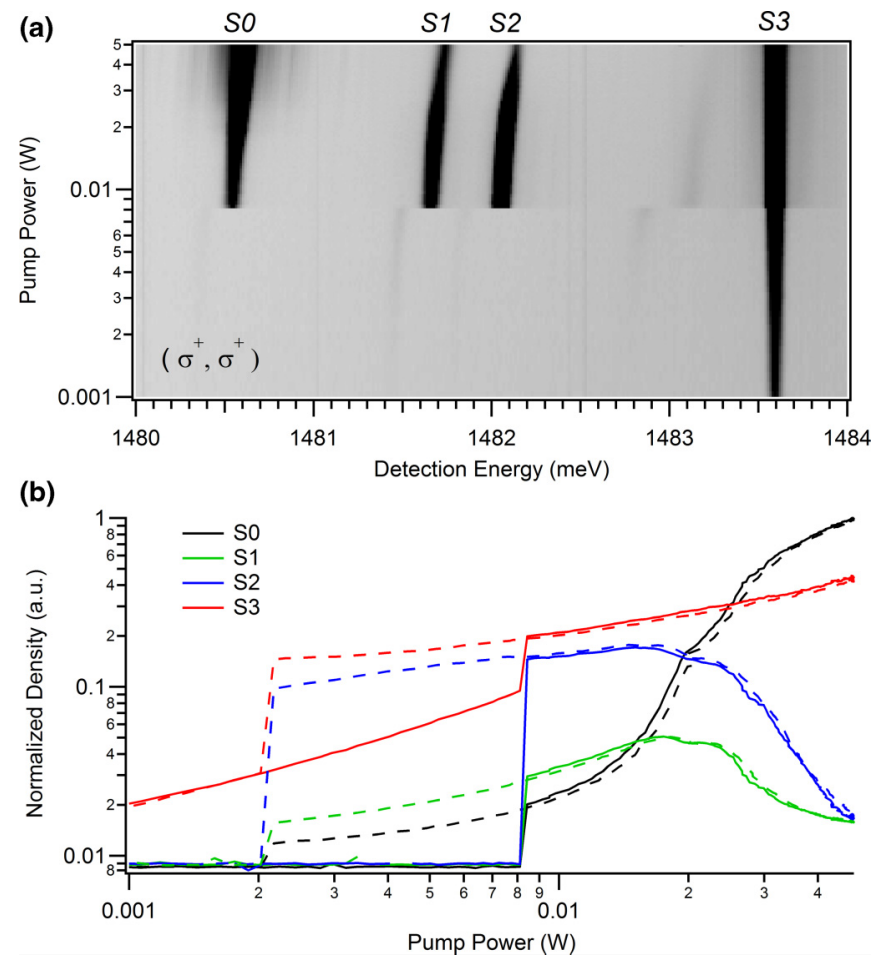

FIG. 2. (Color online) (a) Spectrally resolved intensity of the mesa emission as a function of the pump power (left axis in log scale) when excited $0.3 \mathrm{meV}$ above $S 3$. The sample is excited with $\sigma^{+}$ polarization and the emission is detected for cocircular polarization. The color scale is saturated in order to make the low power zone visible. (b) Integrated and normalized density for the confined states of the mesa as a function of the increasing (solid lines) and decreasing (dashed lines) power. states because of the polariton-polariton interaction. Around $P=8 \mathrm{~mW}, S 3$ gets in resonance with the laser showing an abrupt jump in the intensity due to the more efficient coupling with the laser. The nonlinear jump is then imprinted from $S 3$ to $S 2, S 1$ and $S 0$. The different behavior at the threshold of the lower states depends on the energy separation with respect to $S 3$ and the momentum dispersion, favoring $S 2$. Moreover, the macroscopic occupation of the states is responsible for bosonic stimulation which is activated at $8 \mathrm{~mW}$ when the density threshold is reached in all the lower states. Around $P=20 \mathrm{~mW}$, another nonlinear increase is observed for $S 0$ accompanied by a decrease of the $S 1$ and $S 2$ occupancy. This unusual behavior is the result of the interplay of bosonic stimulated relaxations among the different states. As expected for optical bistability, the opening of the hysteresis loop is observed for $S 3$. This is shown by solid and dashed lines. The presence of a similar hysteresis in the lower energy states is clear evidence of the imprinting of the nonlinearity onto such states. It is important to notice in Fig. 2(a) that, after the first threshold at $P=8 \mathrm{~mW}$, the increase of intensity is followed by a blueshift of all the states. This is due to the sudden increase of the number of particles in the system. Nevertheless, the energy of the states keeps increasing also after the jump showing that we are still in the strong coupling regime and polaritons are still eigenstates of the system.

\section{Onset of coherence}

Another important piece of information can be extracted from the power map of Fig. 2(a) about the onset of coherence in the confined states. It is in fact possible to extract the linewidth as a function of the pump power. This plot is shown in Fig. 3. The emission linewidth of the lower states $S 2-S 0$ narrows at the first threshold $(8 \mathrm{~mW})$, approaching the width of $S 3$ which is imprinted by the laser and limited by the resolution of the spectrometer. In fact, differently from the state $S 3$, in which the coherence is imprinted by the pump laser even at low pump power, the coherence of the states $S 2-S 0$ is the result of the bosonic stimulation due to the crossing of the density threshold in $S 2-S 0$. This spectral narrowing can be interpreted as the onset of macroscopic coherence in the confined states, which behave like polariton lasers $[12,15]$. Around $P=20 \mathrm{~mW}$, due to the interplay of the different bosonic effects, the linewidth of $S 2$ and $S 1$ increases because these states are depopulated in

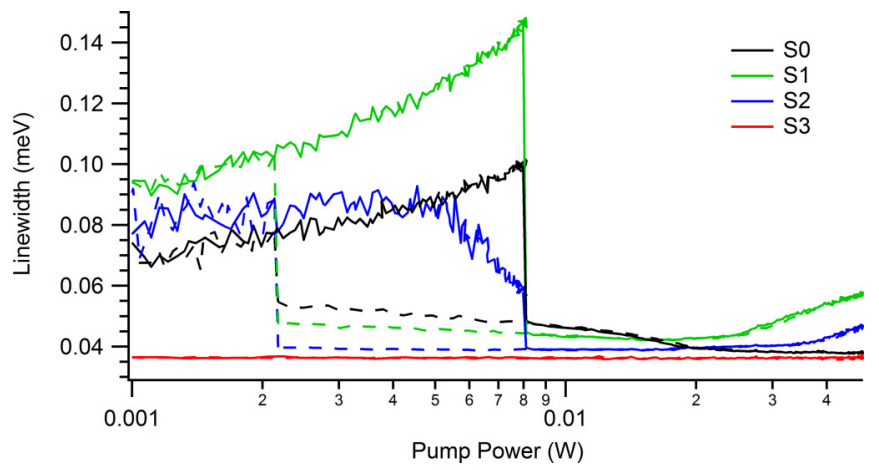

FIG. 3. (Color online) The plot shows the linewidth of all the confined states as a function of the pump power. A narrowing is observed at the onset of the bistability. 
favor of $S 0$ and this reduces their coherence time. On the other hand the coherence time of $S 0$ increases as shown by a further reduction of its linewidth around $P=20 \mathrm{~mW}$. One should stress that such a phenomenon is quite different from a BoseEinstein condensate which is driven by a thermodynamic phase transition. In the thermodynamic picture, the largest occupancy is expected on the lower energy state of the system: the ground state. In our case, we have significant accumulation of particles at different states above the energy of the ground state $S 0$. This phenomenon was already observed in micropillars by means of time resolved measurements [21]. It is important to notice that the linewidth narrowing must be attributed only to the bosonic stimulation and not to the onset of the bistability which is only responsible for an increase of the polariton density in the confined states due to the efficient relaxation mechanism. In fact, without coherence an increase of particle density in a quantum state is usually followed by an increase of the linewidth due to homogeneous broadening. At high density, the increased scattering rate between polaritons reduces the coherence time with a broadening of the linewidth [22].

\section{THEORETICAL MODEL}

The experimental results and interpretations are supported by theory. It is in fact possible to model our system in the framework of the Gross-Pitvaeskii equation, widely used to describe polariton systems [23]. The confined states of the mesa can be treated as separate polariton states coupled through the relaxation processes. A schematic of the polariton states is shown in Fig. 4(a). The coupled equations for the field amplitude of $S 3\left(\psi_{3}\right)$, the density of $S 2\left(n_{2}\right), S 1\left(n_{1}\right)$, and of

(a)

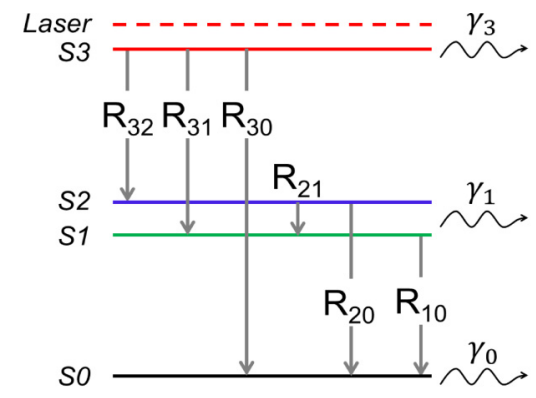

(b)

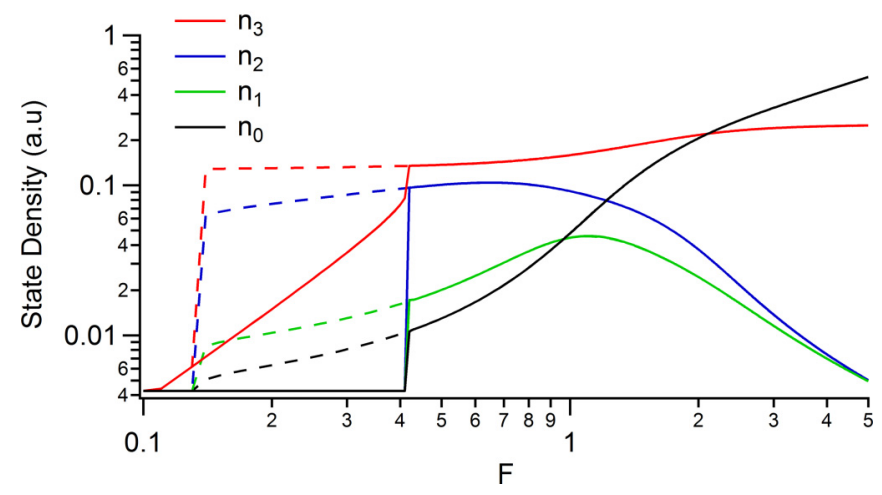

FIG. 4. (Color online) (a) Sketch of the energy levels with the relaxation process $\left(R_{j l}\right)$ and the loss $\left(\gamma_{j}\right)$. (b) Result of the numerical simulations of the density of the confined states coupled through polariton relaxation.
$S 0\left(n_{0}\right)$ read

$$
\begin{aligned}
i \hbar \frac{\partial}{\partial t} \psi_{3}= & {\left[g \sum_{j=0}^{3} n_{j}-\frac{i}{2}\left(\gamma_{3}+\sum_{l=0}^{2} R_{3 l}\left(n_{l}+1\right)\right)\right] \psi_{3}+F e^{-i \delta t} } \\
\frac{\partial}{\partial t} n_{2}= & -\gamma_{2} n_{2}+R_{32}\left(n_{2}+1\right)\left|\psi_{3}\right|^{2}-R_{21}\left(n_{1}+1\right) n_{2} \\
& -R_{20}\left(n_{0}+1\right) n_{2} \\
\frac{\partial}{\partial t} n_{1}= & -\gamma_{1} n_{1}+R_{31}\left(n_{1}+1\right)\left|\psi_{3}\right|^{2}+R_{21}\left(n_{1}+1\right) n_{2} \\
& -R_{10}\left(n_{0}+1\right) n_{1} \\
\frac{\partial}{\partial t} n_{0}= & -\gamma_{0} n_{0}+\sum_{j=1}^{3} R_{j 0}\left(n_{0}+1\right) n_{j}
\end{aligned}
$$

where $\left|\psi_{3}\right|^{2}=n_{3} . g$ is the polariton interaction constant, $\gamma_{j}$ the linewidth, and $R_{j l}$ the phonon scattering rate between the state $j$ and $l . F$ is the pump laser intensity. Bosonic stimulation is considered through the terms $\left(n_{l}+1\right) n_{j}$ [24,25]. In our model we account for the excitonic content of the states by considering a different lifetime for each of them. The values of $\gamma_{j}$ are then rescaled with respect to $\gamma_{0 D}=\frac{\hbar}{30 p s}$ according to the exciton content. In the same fashion, $R_{30}$ and $R_{31}$ are scaled with respect to $R_{32}$ considering the relaxation efficiency among the states in the linear regime extracted from the PLE. Experimentally these are found to be $R_{30}=R_{32} / 1.7$ and $R_{31}=R_{32} / 1.4$. We use $R_{32}=5 \times 10^{-2} \mathrm{meV}, R_{20}=$ $1.2 \times 10^{-4} \mathrm{meV}$, and $R_{21}=R_{10}=0.6 \times 10^{-4} \mathrm{meV}$. Moreover this model takes into account the blueshift of $S 3$ due to polariton interaction inside the same state and among the others with $g=0.01 \mathrm{meV}$. This interaction between different states is introduced due to the spatial overlap between the confined states, in a way similar to the interaction with the excitonic reservoir in the case of nonresonant excited polariton condensates [26]. The simulated densities as a function of the pump laser intensity $F$ are shown in Fig. 4(b). As it happens in the experiments, all states experience an abrupt jump when $S 3$ gets in resonance with the pump laser. The different behavior of $S 2$ with respect to $S 1$ and $S 0$ can be understood considering that the phonon relaxation is more efficient for small energy differences between the initial and the final state. Therefore $S 2$ is favored in this case. At the same time, the increase of the occupancy of the confined states creates the conditions for the onset of bosonic stimulation. By increasing the excitation power the interplay between this effect among all states results in a decrease of $S 2$ and $S 1$ in favor of $S 0$, whose emission gets predominant. The good agreement between simulations and experiments validates our interpretation for the relaxation process and the general physical principles of our model.

\section{CONSERVATION OF THE INITIAL POLARIZATION}

The imprinting of the nonlinearity described in the previous section is enriched by the conservation of the initial polarization (in this specific case $\sigma^{+}$). A power scan similar to Fig. 2(a) is performed filtering out the $\sigma^{+}$component and is displayed in Fig. 5(a). In this case, compared to the one of Fig. 2(a), a different configuration for the detection path 

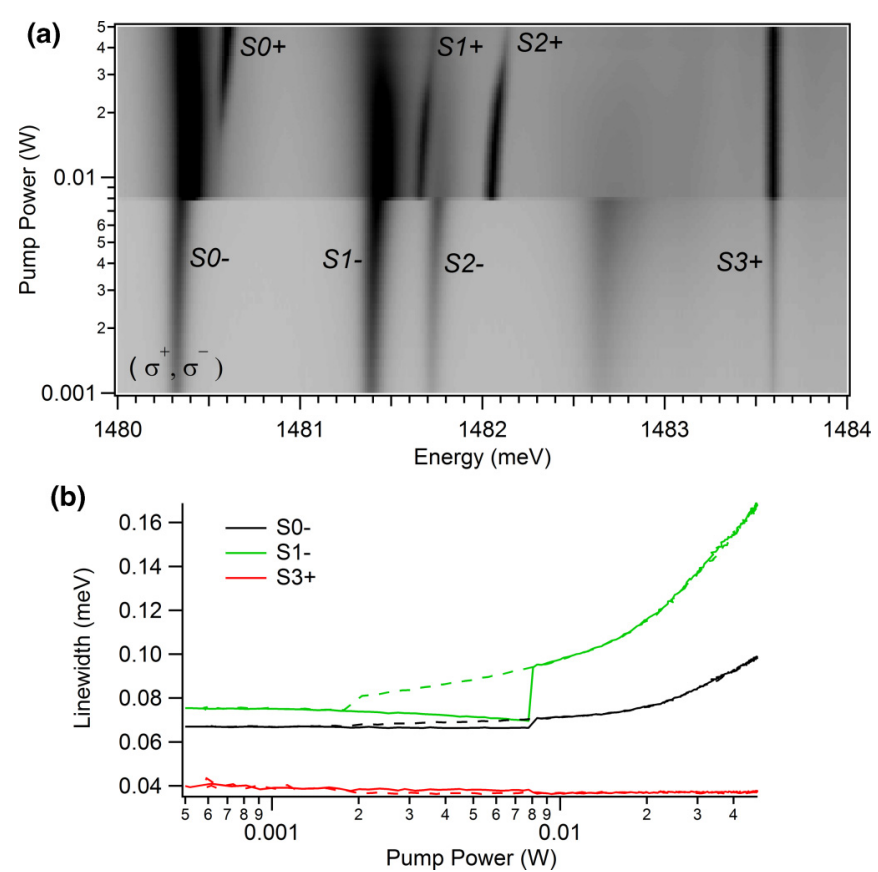

FIG. 5. (Color online) (a) Spectrally resolved intensity map of the mesa emission as a function of the pump power. The sample is excited with $\sigma^{+}$and only $\sigma^{-}$is detected. The color scale of the map is $\sim 400$ times more intense with respect to the case of cocircular polarization detection (Fig. 2(a)). Nevertheless, the emission of $\sigma^{-}$ is so weak compared to the one of $\sigma^{+}$that the latter is still visible in the map. (b) Linewidth of the $\sigma^{-}$emission of the confined states as a function of the pump power.

has been adopted and the color scale is $\sim 400$ times more intense. This scale has been chosen because the $\sigma^{-}$emission is extremely low when compared to $\sigma^{+}$. This observation already tells us that the spin is conserved during the phonon assisted relaxation process. Moreover, the intensity imbalance between the two spin components is so strong that the emission from $\sigma^{+}$is still visible in the power map. Despite this, the impossibility to totally filter out the $\sigma^{+}$helps us to visualize the different behavior of the two spinor emissions. Contrary to $\sigma^{+}$polarized states, whose energies increase while increasing the pump power, the $\sigma^{-}$polaritons do not show any significant blueshift. This can be related to the fact that the polariton population created in $S 3$ is almost completely polarized $\sigma^{+}$. As shown in Fig. 5(b), the $\sigma^{-}$emissions do not experience any narrowing. On the contrary, the linewidth increases as a result of the homogeneous broadening due to the polariton-polariton interaction. In particular, there is a strong increase at $8 \mathrm{~mW}$, namely when the polariton population increases. This observation validates the interpretation about the bosonic stimulation as the driving mechanism for the onset of coherence. Different from the case of the $\sigma^{+}$emission [see Fig. 2(b)] where the polariton density is high enough to trigger bosonic stimulation, for the $\sigma^{-}$emission the density threshold is not crossed and the linewidth keeps increasing also after the bistability threshold. Figure 6 gives an insight about the coexistence of both polarization components in the spectrum for two different powers, $20 \mathrm{~mW}$ (a) and $45 \mathrm{~mW}$ (b). Plot (a) shows the splitting between different polarized components of the same state. It is interesting to observe that the intensity of $S 0^{-}$is stronger than $S 1^{-}$, which is stronger than $S 2^{-}$as well. This can be justified with the assumption that the spin flip mechanism responsible for the appearance of the $\sigma^{-}$emissions is driven by the phonon scattering. Despite the polarization filter, the emission of the $\sigma^{+}$component can be observed. It gets stronger while increasing the pump power and $\mathrm{SO}^{+}$gets comparable to the one of $S 0^{-}$at $P=45 \mathrm{~mW}$. In Fig. 6(b) it is possible to appreciate the difference in linewidth between the two counter-polarized components for the ground state: $\sim 0.1 \mathrm{meV}$ for $\sigma^{-}$and $\sim 0.04 \mathrm{meV}$ for $\sigma^{+}$. For comparison the spectral profiles of the copolarized emission are plotted in dotted lines in Fig. 6. The latter have been extracted from Fig. 2(a) and have been corrected considering the extinction rate of the polarization optics and the different detection configuration. The same experiment has been performed with the pump laser linearly polarized. In this configuration no energy shift is observed between the $\sigma^{+}$and the $\sigma^{-}$emissions because both populations are equally intense.

\section{SELECTIVE POLARITON LASER}

Simple logic operations are achieved by exploiting the unusual emission behavior of the confined polariton states shown in Fig. 2(b). It is possible to define an intensity threshold $\left(I_{t h}\right)$, as shown in the plot on the top of Fig. 7, in order to switch on (1) and off (0) the emission from $S 3, S 2$, and $S 0$ according to the power of the pump laser used as a control beam. The intensity threshold is set such that for $P_{1}=7 \mathrm{~mW}$ only $S 3$ is on. The state $S 2$ and $S 0$ switch on sequentially at $P_{2}=9 \mathrm{~mW}$ and $P_{3}=20 \mathrm{~mW}$. Eventually at $P_{4}=45 \mathrm{~mW}, S 2$ turns off due
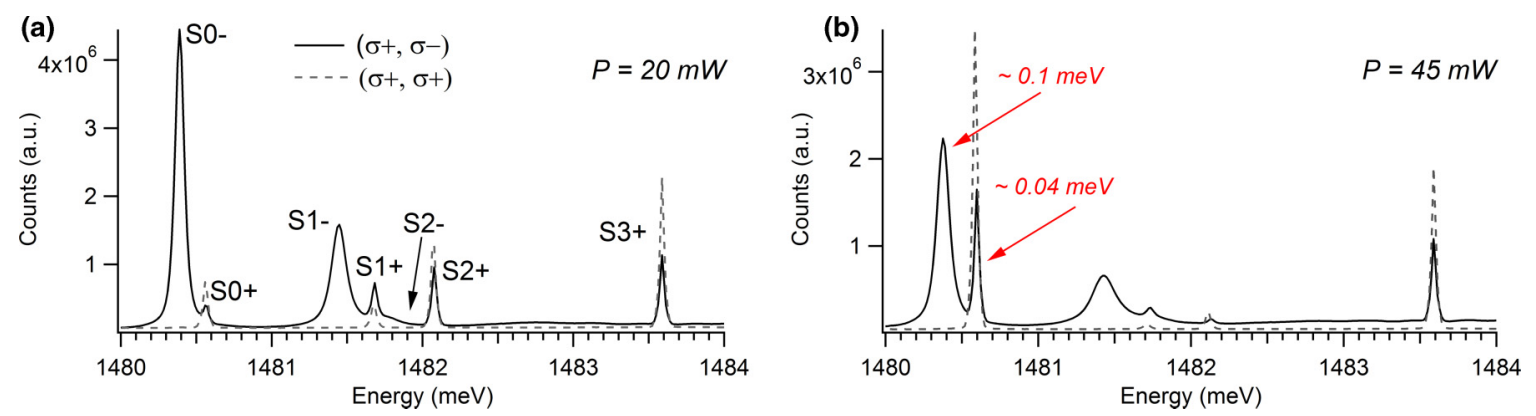

FIG. 6. (Color online) Spectral emission of the mesa for counter-polarized (solid line) and copolarized (dotted line) detection at $P=20 \mathrm{~mW}$ (a) and $P=45 \mathrm{~mW}$ (b). The spectra for copolarized emission are corrected considering the extinction rate of the polarization optics and the different detection configuration. 


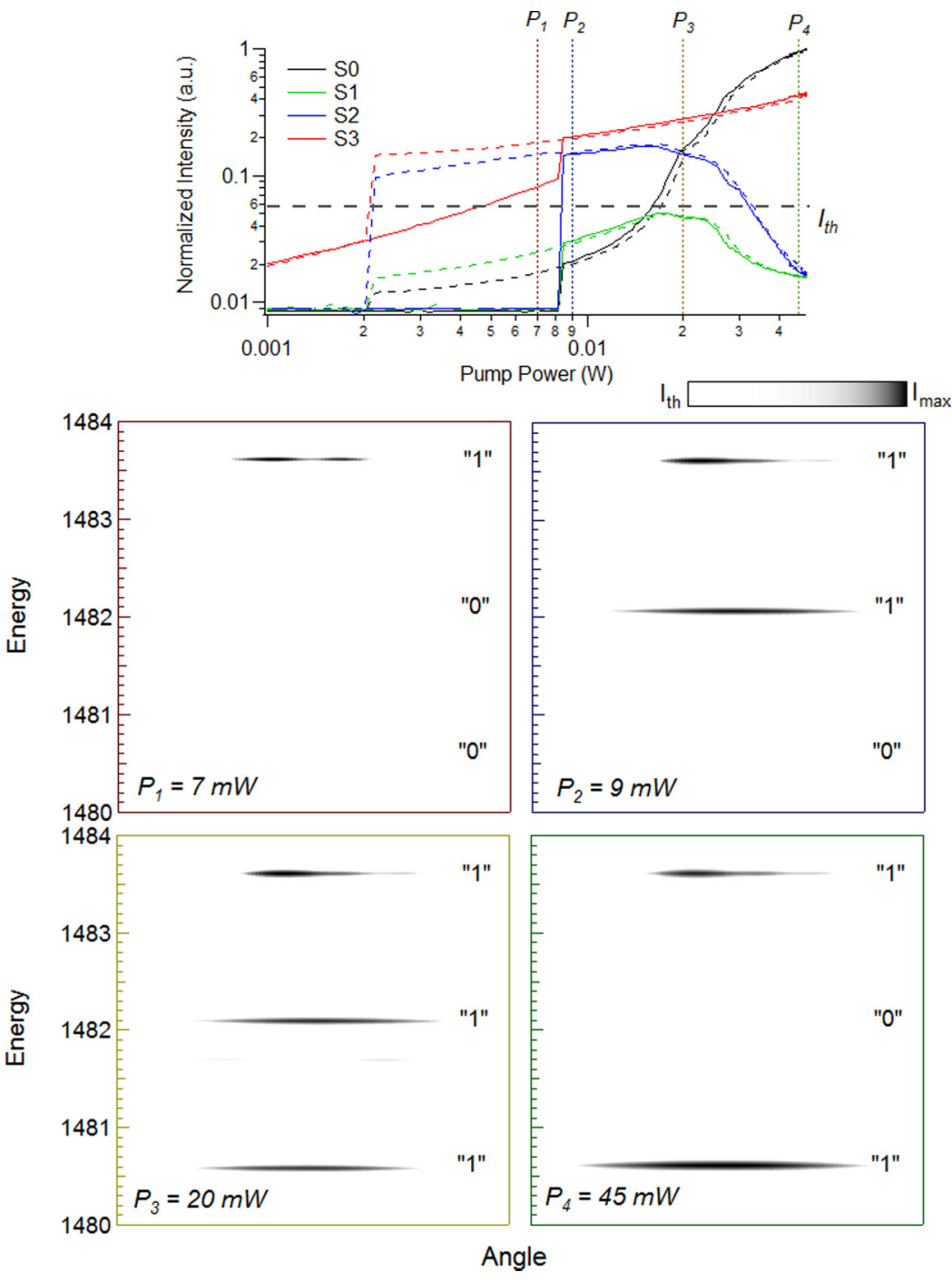

FIG. 7. (Color online) The plot on the top shows the integrated intensity of the confined states. The intensity threshold for the switch $\left(I_{t h}\right)$ and the powers at which this occurs for the different states are stressed by dashed and vertical dotted lines. The panels in the bottom show the experimental dispersion of the mesa for different powers of the control beam. The emission of three states can be controlled (0-1) by tailoring the input power. A color scale minimum is set in order to simulate an intensity threshold for the switch.

to bosonic stimulation. The panels of Fig. 7 are the dispersions of the confined lower polaritons for different excitation powers showing the lasing emission at different energy of the polariton confined states.

The control on the polarization of the input beam allows doubling of the available logic operations considering the two projections of the spin up and spin down states. The initial laser beam, tailored in intensity and polarization, impinges on the polychromatic polariton laser switch. As a function of the incident beam state, different outputs can be produced and detected, for example, using a polarized beam splitter linked to sets of wavelength demultiplexers. If we consider the polariton spin, nine independent configurations can be achieved by controlling the activation of the lasing from $S 3, S 2$, and $S 0$. These laser emissions at different energy could be used to trigger the activation of other selective polariton lasers engineered such that in a mesa network the highest confined states of the other mesas match the emission energy of the first. The speed at which the output signal can be modulated is governed by the phonon assisted relaxation rate which is estimated to be on the order of tens of GHz. This rate will be increased in the perspective of working at higher temperature due to the enhancement of phonon-polariton scattering rate [27].
Extended logic operations can be achieved by setting $I_{t h}$ such that the state $S 1$ is included as an extra forth channel. Considering the spin, this choice adds four logic combinations in the working principle of the selective laser.

\section{CONCLUSIONS}

We have discussed the relaxation mechanisms of polaritons confined inside a $3 \mu \mathrm{m}$ diameter mesa. In particular we have investigated the properties of a mesa at a rather positive detuning, which shows peculiar features regarding the exciton content for the different confined states. The properties of such a mesa are studied by means of photoluminescence excitation techniques in the linear regime, showing that efficient relaxation from the state $S 3$ occurs toward lower energy states. The reason for this effect is attributed to the large excitonic content that provides a long lifetime for polariton which thus have time to interact with a bath of acoustic phonons and relax down the trap. We have also shown that this process can be used to imprint the nonlinearity occurring in $S 3$ onto the lower states, creating the conditions for polariton lasing from the different confined states of the mesa. In particular this is achieved by generating a bistable optical 
effect on the $S 3$. The increasing number of particles in the system allows for the onset of bosonic stimulation among the states which is accompanied by spectral narrowing of all the polariton emissions. This clue can be interpreted as the beginning of phase coherence in the system. We have also observed a conservation of the initial spin which results in the conservation of the original polarization of the control laser beam. The combination of these two properties leads to the realization of a device based on polariton lasing at different wavelengths. By tuning the power of the control beam, logic operations (ON-OFF) can be achieved by switching the laser emission from the single confined states.

\section{ACKNOWLEDGMENTS}

The authors thank P. Lugan and I. Carusotto for fruitful discussions. The authors acknowledge founding from SNF under project 135003 and European Research council under project N219120. M.W. acknowledges financial support from the FWO Odysseus program.
[1] D. A. B. Miller, D. S. Chemla, T. C. Damen, A. C. Gossard, W. Wiegmann, T. H. Wood, and C. A. Burrus, Appl. Phys. Lett. 45, 13 (1984).

[2] G. Grosso, J. Graves, A. T. Hammack, A. A. High, L. V. Butov, M. Hanson, and A. Gossard, Nat. Photon. 3, 577 (2009).

[3] G. Grosso, G. Nardin, F. Morier-Genoud, Y. Léger, and B. Deveaud-Plédran, Phys. Rev. B 86, 020509 (2012).

[4] A. Baas, J. P. Karr, H. Eleuch, and E. Giacobino, Phys. Rev. A 69, 023809 (2004).

[5] T. Paraïso, M. Wouters, Y. Léger, F. Morier-Genoud, and B. Deveaud-Plédran, Nat. Mater. 9, 655 (2010).

[6] C. Anton, T. Liew, G. Tosi, M. Martin, T. Gao, Z. Hatzopoulos, P. Eldridge, P. Savvidis, and L. Vina, Appl. Phys. Lett. 101, 261116 (2012).

[7] M. Steger, C. Gautham, B. Nelsen, D. Snoke, L. Pfeiffer, and K. West, Appl. Phys. Lett. 101, 131104 (2012).

[8] D. Ballarini, M. De Giorgi, E. Cancellieri, R. Houdre, E. Giacobino, R. Cingolani, A. Bramati, G. Gigli, and D. Sanvitto, Nat. Commun. 4, 1778 (2013).

[9] R. Cerna, Y. Léger, T. K. Paraïso, M. Wouters, F. MorierGenoud, M. T. Portella-Oberli, and B. Deveaud, Nat. Commun. 4, 2008 (2013).

[10] A. Amo, T. C. H. Liew, C. Adrados, R. Houdré, E. Giacobino, A. V. Kavokin, and A. Bramati, Nat. Photon. 4, 361 (2010).

[11] J. Kasprzak, M. Richard, S. Kundermann, A. Baas, P. Jeambrun, J. M. J. Keeling, F. M. Marchetti, M. H. Szymańska, R. Andre, J. L. Staehli, V. Savona, P. B. Littlewood, B. Deveaud, and Le Si Dang, Nature (London) 443, 409 (2006).

[12] H. Deng, G. Weihs, D. Snoke, J. Bloch, and Y. Yamamoto, Proc. Natl. Acad. Sci. USA 100, 15318 (2003).

[13] C. Schneider, A. Rahimi-Iman, N. Y. Kim, J. Fischer, I. G. Savenko, M. Amthor, M. Lermer, A. Wolf, L. Worschech, V. D. Kulakovskii, I. A. Shelykh, M. Kamp, S. Reitzenstein,
A. Forchel, Y. Yamamoto, and S. Höfling, Nature (London) 497, 348 (2013).

[14] S. Christopoulos, G. B. H. von Högersthal, A. J. D. Grundy, P. G. Lagoudakis, A. V. Kavokin, J. J. Baumberg, G. Christmann, R. Butté, E. Feltin, J.-F. Carlin, and N. Grandjean, Phys. Rev. Lett. 98, 126405 (2007).

[15] D. Bajoni, P. Senellart, E. Wertz, I. Sagnes, A. Miard, A. Lemaître, and J. Bloch, Phys. Rev. Lett. 100, 047401 (2008).

[16] R. I. Kaitouni, O. El Daï, A. Baas, M. Richard, T. Paraiso, P. Lugan, T. Guillet, F. Morier-Genoud, J. D. Ganière, J. L. Staehli, V. Savona, and B. Deveaud, Phys. Rev. B 74, 155311 (2006).

[17] P. Lugan, D. Sarchi, and V. Savona, Phys. Status Solidi C 3 , 2428 (2006).

[18] G. Nardin, Y. Léger, B. Pietka, F. Morier-Genoud, and B. Deveaud-Plédran, Phys. Rev. B 82, 045304 (2010).

[19] T. K. Paraïso, D. Sarchi, G. Nardin, R. Cerna, Y. Leger, B. Pietka, M. Richard, O. El Daï, F. Morier-Genoud, V. Savona, and B. Deveaud-Plédran, Phys. Rev. B 79, 045319 (2009).

[20] N. A. Gippius, I. A. Shelykh, D. D. Solnyshkov, S. S. Gavrilov, Y. G. Rubo, A. V. Kavokin, S. G. Tikhodeev, and G. Malpuech, Phys. Rev. Lett. 98, 236401 (2007).

[21] M. Maragkou, A. J. D. Grundy, E. Wertz, A. Lemaître, I. Sagnes, P. Senellart, J. Bloch, and P. G. Lagoudakis, Phys. Rev. B 81, 081307 (2010).

[22] D. Porras and C. Tejedor, Phys. Rev. B 67, 161310 (2003).

[23] I. Carusotto and C. Ciuti, Rev. Mod. Phys. 85, 299 (2013).

[24] F. Tassone and Y. Yamamoto, Phys. Rev. B 59, 10830 (1999).

[25] F. Tassone and Y. Yamamoto, Phys. Rev. A 62, 063809 (2000).

[26] E. Wertz, L. Ferrier, D. D. Solnyshkov, R. Johne, D. Sanvitto, A. Lemaître, I. Sagnes, R. Grousson, A. V. Kavokin, P. Senellart, G. Malpuech, and J. Bloch, Nat. Phys. 6, 860 (2010).

[27] R. P. Stanley, S. Pau, U. Oesterle, R. Houdré, and M. Ilegems, Phys. Rev. B 55, R4867 (1997). 